\title{
Relation between Mental Health and Suicide Resilience Among University Students in South Korea
}

\author{
Seong-Eun Kim \\ Dept. of Nurisng, Woosuk University, Republic of Korea \\ fromutos@daum.net
}

\begin{abstract}
This study used convenience sampling and self-reported surveys to investigate the mental health status and suicide resilience of 297 university students in South Korea. Significant associations were found between suicide resilience and the mental health index $(\beta=0.50, p<.001)$. The enhancement of university students' psychosocial functions should promote suicide prevention and increase mental health.
\end{abstract}

Keywords: Mental, Health, Suicide, Resilience, Student, Survey

\section{Introduction}

University students, usually in their twenties, are highly educated people who are important, productive resources for society. Their suicide is a very serious problem because it brings about loss of life, physical damage, medical cost, sorrow, pain, and huge financial loss in terms of dissolution of families and local communities [1][2]. Each suicide affects 6 to 18 families, friends, and other related people [3][4]. Therefore, it is necessary to identify the mental health issues of university students to prevent suicide.

Life is full of hardship and adversity. It requires potential to turn the confronted challenges and difficulties into stepping-stones for the future [5]. Resilience refers to the capability to overcome difficulties, adjust oneself to the circumstances, and make mental progress [6]. Suicide resilience refers to the capability to efficiently overcome the crisis of suicide and flexibly deal with the situation [7]. Suicide resilience enables one to overcome and withstand risky situations and to suppress suicidal impulses; it is a powerful protective factor during depression [8]. Therefore, strengthening suicide resilience is critical to prevent suicidal ideation as higher resilience has been reported to be associated with positive mental health [9].

There is not a definite boundary between mental health and mental illness; rather, there is a spectrum of healthy/unhealthy and adaptive/maladaptive reactions to disease [10][11][12]. Most interventions focus on an individual's reaction to a potential or real health problem. Keyes [13] suggested that mental health and mental illness are not located on opposite poles; rather, they form independent, continuous lines, which are more appropriate for the complete mental health model where mental illness and mental well-being are correlated with each other, but exist as independent dimensions [12][13].This classification of subcategories implies that a person of genuine mental health is free from mental illness and experiencing positive mental well-being at the same time [11].

Therefore, the specific objectives were as follows:

1) To investigate the status of mental health and suicide resilience of university students.

Article history:

Received (August 19, 2016), Review Result (September 21, 2016), Accepted (October 25, 2016) 
2) To verify the correlation between the mental health index and suicide resilience

\section{Methods}

\subsection{Study design}

This descriptive, survey research investigated the degree of mental health and suicide resilience in university students to identify the relationship between mental health and suicide resilience.

\subsection{Participants}

The participants were 297 students from universities located in two (Chungcheong and Honam provinces) of five regions in South Korea.

\subsection{Questionnaire}

\subsubsection{Mental health index}

The Korean MHI (K-MHI), which is a single index integrating information about mental illness and mental well-being, is calculated by the equation: "(mental well-being score) $/ 2+$ (100-mental illness score)/2" [14]. The mental well-being score is $100 *($ percentage of mental well-being score), whereas the mental illness score is $100^{*}$ (percentage of mental illness score). The percentage of mental well-being score (K-MHC-SF) is the value obtained by dividing the total well-being score by the maximum well-being score of 70 , while the percentage of mental illness total score is the value obtained by dividing the total mental illness score by the maximum mental illness score of 52 . The range of the MHI is from 0 to 100, with higher scores representing better mental health.

The Korean Mental Disorder Inventory (K-MDI) developed by Lim et al. [14] was used to measure mental illness. This 14-item self-reported inventory measured the presence of mental illness (13 items) using a five-point Likert scale ranging from 1="strongly disagree" to $5=$ "strongly agree" and social functions difficulty $(1 \mathrm{item})$ if a participants responded to one of the previous questions with a 4 or 5 . The total score was calculated by summing the first 13 questions. If a participant responded to at least one of the 13 questions about mental illness with "agree" or "strongly agree" and responded that he or she had experienced a social functions difficulty due to mental illness, the participant was classified as having a mental illness. The reliability of the inventory in this study was $\alpha=0.93$.

Mental well-being was measured using the Korean Mental Health Continuum short-form (K-MHC-SF) [14] by validating the Mental Health Continuum-Short Form (MHC-SF) that was developed by Keyes et al. [15]. The K-MHC-SF included 14 questions with three subscales: emotional well-being, psychological well-being, and social well-being. For each question, the frequency of experiencing mental well-being during the last month was measured using a six-point Likert scale ranging from 1="never" to 6="daily." Mental wellbeing was categorized as "flourishing," "moderate," or "languishing." The "flourishing" category included participants who responded that they experienced emotional well-being related to at least one of the three questions and they experienced psychological well-being and social well-being related to at least six of the 11 questions as "daily" or "almost daily." The "languishing" category included participants who responded to the same scales as either "never" or "once or twice a month." Participants who did not fit either previous category were 
allocated to the "moderate" category. The original inventory and this study's inventory had reliabilities of $\alpha=0.89$ and $\alpha=0.87$, respectively.

\subsubsection{Suicide resilience}

Suicide resilience was measured by using the Suicide Resilience Inventory-Korean Version, which was prepared by Noh, Chang, and Kim [16] by standardizing the Suicide Resilience Inventory developed by Osman et al. [7] to assess factors that protect suicidal ideation and behavior. The inventory included 19 questions including seven "internal protective" questions measuring beliefs or emotions relevant to one's self and life satisfaction, five "external protective" questions measuring the ability to find perceived external sources that might help during difficult times, and seven "emotional stability" questions measuring the positive belief to control one's own thoughts and behaviors related to suicide when under stress. For each question, suicide resilience was measured using a 6-Likert scale ranging from 1="strongly disagree" to $6=$ "strongly agree" with higher scores representing a lower degree of suicide risk. The reliability in Noh and colleagues' original study and in this study were both Cronbach's $\alpha=0.94$.

\subsection{Data analysis}

All data were analyzed using IBM SPSS for Windows, version 22.0 program. The correlation between mental health index and suicide resilience was analyzed using Pearson's correlation coefficient. The statistical significance level was set at $\mathrm{p}$-value $<.05$.

\section{Results}

\subsection{Mean score of mental health and suicide resilience}

The mean score of the mental health index and suicide resilience were $65.68 \pm 11.80$ and $4.62 \pm 0.64$. The mean score for the three suicide resilience subcategories, which were "internal protective," "external protective," and "emotional stability," were $4.30 \pm 0.76,4.76$ \pm 0.73 , and $4.83 \pm 0.75$, respectively [Table 1].

Table 1. Mean score of mental health and suicide resilience $(\mathrm{N}=297)$

\begin{tabular}{|c|c|c|c|}
\hline Variables & M SD & Min & Max \\
\hline Suicide resilience & $4.62 \pm 0.64$ & 2.47 & 6.00 \\
\hline Internal protective & $4.30 \pm 0.76$ & 1.71 & 6.00 \\
\hline External protective & $4.76 \pm 0.73$ & 1.40 & 6.00 \\
\hline Emotional stability & $4.83 \pm 0.75$ & 2.43 & 6.00 \\
\hline Mental health index & $65.68 \pm 11.80$ & 30.60 & 97.50 \\
\hline
\end{tabular}

Note. $M=$ mean; $S D=$ standard deviation.

\subsection{Correlations for suicide resilience and mental health index}

A positive correlation was found between the mental health index and the suicide resilience level $(\mathrm{r}=.68, \mathrm{p}<.001)$ [Table 2]. 
Table 2. Correlations for suicide resilience and mental health $(\mathrm{N}=297)$

\begin{tabular}{|c|c|c|c|c|c|}
\hline \multirow{2}{*}{ Variables } & SR & IP & EP & ES & MHI \\
\hline & $\mathrm{r}(p)$ & $\mathrm{r}(p)$ & $\mathrm{r}(p)$ & $\mathrm{r}(p)$ & $\mathrm{r}(p)$ \\
\hline Suicide resilience & 1 & & & & \\
\hline Internal protective & $\begin{array}{c}.85 \\
(<.001)\end{array}$ & 1 & & & \\
\hline External protective & $\begin{array}{c}.84 \\
(<.001)\end{array}$ & $\begin{array}{c}.61 \\
(<.001)\end{array}$ & 1 & & \\
\hline Emotional stability & $\begin{array}{c}.86 \\
(<.001)\end{array}$ & $\begin{array}{c}.53 \\
(<.001)\end{array}$ & $\begin{array}{c}.63 \\
(<.001)\end{array}$ & 1 & \\
\hline Mental health index & $\begin{array}{c}.68 \\
(<.001)\end{array}$ & $\begin{array}{c}.73 \\
(<.001)\end{array}$ & $\begin{array}{c}.53 \\
(<.001)\end{array}$ & $\begin{array}{c}.45 \\
(<.001)\end{array}$ & 1 \\
\hline Mental illness & $\begin{array}{c}-.45 \\
(<.001)\end{array}$ & $\begin{array}{c}-.42 \\
(<.001)\end{array}$ & $\begin{array}{c}-.35 \\
(<.001)\end{array}$ & $\begin{array}{c}-.37 \\
(<.001)\end{array}$ & $\begin{array}{c}-.75 \\
(<.001)\end{array}$ \\
\hline Mental health continuum & $\begin{array}{c}.62 \\
(<.001)\end{array}$ & $\begin{array}{c}.72 \\
(<.001)\end{array}$ & $\begin{array}{c}.49 \\
(<.001)\end{array}$ & $\begin{array}{c}.36 \\
(<.001)\end{array}$ & $\begin{array}{c}.85 \\
(<.001)\end{array}$ \\
\hline
\end{tabular}

Note. $S R=$ suicide resilience; $I P=$ internal protective; $E P=$ external protective; $E S=$ emotional stability; $M H I=$ mental health index

\section{Discussion}

This study investigated the mental health status and suicide resilience of Korean university students and identified the correlation between mental health and suicide resilience. The mean of mental health index score was 65.68 points, which was partially consistent with Lim and colleagues [14] indicating that the overall mental health of university students is moderate. However, the suicide resilience score was low, which was similar to the suicide resilience score (4.62) found in another study conducted with university students in Korea [16]. On the contrary, in previous studies [7][17] conducted in the U.S. with university students and adults aged between 20 and 25 years the suicide resilience score ranged from 5.3 to 5.5. Perhaps the resilience score of Korean students is lower because the score in the "internal protective" subcategory is particularly low, indicating that the result may be correlated with the low "Happy Planet Index" of Koreans [18].

A positive correlation was found between the mental health index and the suicide resilience level. The mental health index, which covers mental illness and mental well-being together, becomes higher when the mental illness level becomes lower and the mental well-being level becomes higher. Therefore, the principal method of preventing and overcoming suicides in Korea may need to be reconsidered. Currently in Korea, the emphasis is put on the early detection and the thorough and intensive treatment of mental illnesses based on the findings that $62.2 \%$ of those who attempt to commit suicide in Korea have psychiatric symptoms and many of the suicide attempters who deny having psychiatric symptoms may actually have one [19]. However, this study suggests that to prevent suicides and strengthen suicide resilience, efforts need to be made in the early detection and treatment of mental illness, the controlling of the risk factors through a psychopathological approach, and in the promotion of mental well-being by improving psychosocial functions.

\section{Conclusions}

In this study, the relation between mental health and suicide resilience of university students in South Korea was verified. A positive correlation was found between the mental 
health and the suicide resilience. Therefore, for the future improvement of suicide resilience, an approach that includes the prevention and treatment of mental illness and the promotion of mental well-being should be actively considered. However, the present study is limited because the participants were selected by convenience sampling; therefore, they do not represent the entire population. Therefore, a replicated and extended study should be conducted to reflect all university students in Korea. Additionally, a study examining the elderly, which have the highest suicide rate in South Korea, should be conducted.

\section{References}

[1] A. Marschean and I.A. Kincaid, "Developing a plan and strategy for suicide prevention in the commonwealth," (Senate Document No.19), Richmond, Virginia: The Joint Commission, (2003)

[2] Park (2013), "Development and evaluation of a crisis intervention program for suicide attempters," Journal of Korean Academy of Psychiatric and Mental Health Nursing, vol.22, no.4, pp.368-376, (2013)

[3] C.W. Drapeau and J.L. Mcintosh., "U.S.A. Suicide: 2014 official final data," Washington, DC: American Association of Suicidology, (2015)

[4] A.J. Knieper, "The suicide survivor's grief and recovery," Suicide and Life-threatening Behavior, vol.29, no.4, pp.353-364, (1999) DOI:10.1111/j.1943-278x.1999.tb00530.x

[5] J.H. Kim, "Resilience," Kyung-gi: Wisdomhouse Inc., (2011)

[6] E.J. Anthony, "Risk, vulnerability, and resilience: An overview," In E. J. Anthony \& B. J. Cohler (Eds.), The invulnerable child, New York, NY: Guilford Press, pp.3-48, (1987)

[7] A. Osman, P.M. Gutierrez, J.J. Muehlenkamp, F. Dix-Richadson, F.X. Barrios, and B.A. Kopper, "Suicide resilience inventory-25: Development and preliminary psychometric properties," Psychological Reports, vol.94, no.3, pp.1349-1360, (2004) DOI:10.2466/PR0.94.3.1349-1360

[8] S.Y. Ahn, J.H. Kim and B.Y. Choi, "Meta-analysis on variables related to suicidal ideation among college students," The Korean Journal of School Psychology, vol.12, no.3, pp.385-405, (2015)

[9] Y.M. Han, "The relation between college students' self-resilience and mental health," Journal of Human Understanding and Counseling, vol.30, no.1, pp.97-110, (2009)

[10] K.J. Lee, J S. Won, S.B. IM, S.A. Kim, S.J. Kim, and S. Lee, "Psychiatric-mental health nursing (6th ed.)," Seoul: Soomoonsa, (2011)

[11] C.L. Keyes, "Mental illness and/or mental health? Investigating axioms of the complete state model of health," Journal of Consulting and Clinical Psychology, vol.73, no.3, pp.539-548, (2005) DOI:10.1037/0022-006X.73.3.539

[12] C.L. Keyes and S.J. Lopez, “Toward a science of mental health,” In C.R. Snyder and S.J. Lopez (Eds.), Handbook of positive psychology, New York, NY: Oxford University Press, pp.45-59, (2002)

[13] G.J. Westerhof and C.L. Keyes, "Mental illness and mental health: The two continua model across the lifespan,” Journal of Adult Development, vol.17, no.2, pp.110-119, (2010)

[14] Y.J. Lim, Y.G. Ko, H.C. Shin, and Y. Cho, "The mental health in South Koreans," Psychology toward happiness: 2010 August 19-21, Seoul National University, Seoul, pp.233-257, (2010)

[15] C.L. Keyes, M. Wissing, J.P. Potgieter, M. Temane, A. Kruger and S. Van Rooy, "Evaluation of the mental health continuum-short form (MHC-SF) in Setswana-speaking south africans," Clinical Psychology and Psychotherapy, vol.15, no.3, pp.181-192, (2008) DOI:10.1002/cpp.572

[16] J.H. Noh, S.J. Chang and S.E. Kim, "Validity and reliability of the suicide resilience inventory-Korean version for Korean university students," Journal of the Korea Academia-Industrial cooperation Society, vol.17, no.1, pp.508-517, (2016)

[17] C. Wang, "Depression and suicide behavior among college students: Understanding the moderator effects of self-esteem and suicide resilience," Ph.D. dissertation, University of Denver, Denver, (2013) DOI:10.1080/00193089.1960.10534087 
Relation between Mental Health and Suicide Resilience Among University Students in South Korea

[18] J.F. Helliwell, R. Laryard, and J. Sachs (Eds.), "World happiness report 2016," Update New York, NY: Sustainable Development Solutions Network, vol.1, pp.18-22, (2016)

[19] Ministry of Health \& Welfare, “Annual report 2013: National survey on suicide,” Sejong, (2014) 